\title{
REMARK ON .SOME INTEGRALS INVOLVING PRODUCTS OF WHITTAKER FUNCTIONS ${ }^{1}$
}

\author{
H. M. SRIVASTAVA
}

\begin{abstract}
It is observed that the literature contains erroneous formulas for infinite integrals involving the product of two Whittaker functions. For instance, the main result, involving Meijer's $G$-function, of K. L. Arora and S. K. Kulshreshtha's paper in these Proceedings and all its particular cases may be cited.
\end{abstract}

1. L. J. Slater states $[2$, p. $56,(3.7 .17)]$

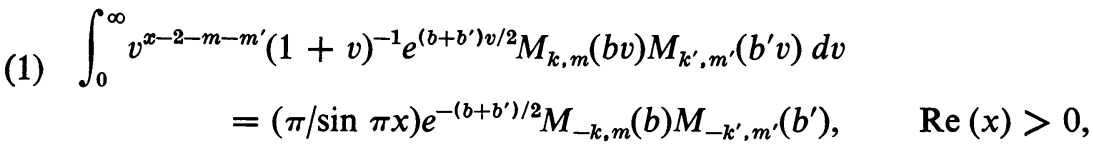
where $M_{k, m}(x)$ denotes the Whittaker function.

Her proof of this formula makes use of the $\Gamma$-function integral

$$
\int_{0}^{\infty} v^{x-1}(1+v)^{-y} d v=\Gamma(x) \Gamma(y-x) / \Gamma(y),
$$

which is valid for $0<\operatorname{Re}(x)<\operatorname{Re}(y)$. She applies it, however, without satisfying the condition $\operatorname{Re}(x)<\operatorname{Re}(y)$. Therefore the proof of (1) is invalid and indeed the result is not true. If, for instance, $b$ and $b^{\prime}$ are positive, the integrand in (1) increases exponentially as $v \rightarrow \infty$, since

$$
M_{k, m}(x) \sim C(k, m) x^{-k} e^{x / 2} \quad(x \rightarrow \infty),
$$

where $C(k, m)$ is a constant depending upon $k$ and $m$.

Similar remarks would apply equally well to Slater's main formula (3.7.9) and its other special cases (3.7.10) through (3.7.13) in [2, pp. 55-56].

2. It may be of interest to observe that the recent formulas, involving Meijer's $G$-function, given by K. L. Arora and S. K. Kulshreshtha in [1]

Received by the editors December 4, 1970 and, in revised form, February 22, 1971. AMS 1970 subject classifications. Primary 30A86, 33A35, 44A20; Secondary 33A15, 33A30, 33A70.

Key words and phrases. Infinite integral, Whittaker functions, Meijer's G-function, gamma function, asymptotic expansions, special functions.

${ }^{1}$ Supported in part by NRC Grant A-7353. 
are based on the integral (1) and are therefore incorrect, at least for some values of the parameters involved. This can easily be verified by considering the well-known asymptotic expansions of the various special functions involved in the infinite integrals evaluated in [1]. The details are, therefore, omitted.

\section{REFERENCES}

1. K. L. Arora and S. K. Kulshreshtha, An infinite integral involving Meijer Gfunction, Proc. Amer. Math. Soc. 26 (1970), 121-125. MR 41 \#5665.

2. L. J. Slater, Confluent hypergeometric functions, Cambridge Univ. Press, New York, 1960. MR 21 \#5753.

Department of Mathematics, University of Victoria, Victoria, British Columbia, Canada 